\title{
FLUJO DE FONDOS: PODEROSA HERRAMIENTA DE GESTIÓN EN LOS NEGOCIOS (Parte I)
}

\author{
JOSÉ PORLLES LOARTE* \\ E-mail: joseporlles@yahoo.com
}

\begin{abstract}
RESUMEN
Se evidencia dificultades en el uso del Estado de Movimiento de Fondos, en la medida que en la variada literatura no se clarifica -con la contundencia del caso- la existencia de tres enfoques de definición de fondos, que se adoptan en función del objetivo que se persiga en el análisis. Esta situación conduce a que dicho Estado sea el menos comprendido y por tanto no se haya amplificado su uso efectivo en las decisiones financieras. Considerando que las herramientas metodológicas establecen una secuencia de pautas de acción para afrontar un determinado tipo de problemas, el presente trabajo tiene la finalidad de mejorar de manera integral la calidad del análisis e interpretación del movimiento de fondos en sus diferentes versiones, para determinar cambios significativos en un periodo determinado de la gestión. En esta primera parte se establece el marco conceptual del flujo de fondos para su cabal comprensión y utilización como una sólida herramienta de gestión.
\end{abstract}

Palabras claves: Fondos, flujo de fondos, flujo de efectivo, fondo de maniobra, estructura patrimonial.

\begin{abstract}
One demonstrates difficulties in the use of the state of movement of bottoms, in the measurement that in varied Literature does not clarify with the forcefulness of the case the existence of three approaches of definition of bottoms that are adopted based on the objective that is persecuted in the analysis. This situation leads to that this state is less included/ understood and therefore its effective use in the financial decisions has not been amplified. Considering which the methodologic tools establish a sequence of action guidelines to confront a certain kind of problems, the present work has this context with the purpose of improving of integral way the quality in the analysis and interpretation of the movement of bottoms in its different versions to determine the significant changes in a determined period of the management. In this first part settles down the conceptual frame of the flow of bottoms for its exact understanding and use like a solid tool of management.
\end{abstract}

Keywords: Bottoms, flow of bottoms, cash flow, bottom of maneuver, patrimonial structure.

* Magíster en Administración. Profesor Asociado del Departamento de Análisis y Diseño de Procesos de la UNMSM. 


\section{INTRODUCCIÓN}

La información financiera que obligadamente deben presentar las empresas a sus accionistas en las Memorias Anuales, así como a la SUNAT, banqueros, inversionistas, ejecutivos de la empresa y acreedores en general, cubre los estados financieros siguientes:

- El Balance General (BG).

- El Estado de Ganancias y Pérdidas (EPG).

- La Situación Patrimonial.

- El Estado de Flujos de Efectivo o Estado Movimiento de Fondos.

De estos reportes periódicos el menos comprendido y usado correctamente es el Estado de Movimiento de Fondos, a pesar que representa una herramienta sumamente útil en el análisis y gestión empresarial.

Es preciso anotar que existen diversos términos para identificar este documento y cada uno de ellos se adecúa a determinados propósitos, lo que tal vez dificulta su manejo eficaz. Así tenemos que recibe varios nombres en la variada literatura financiera:

- Estado de Fuentes y Usos de Fondos.

- Estado de Flujo de Fondos.

- Estado de Flujos de Efectivo (o Flujo de Caja).

- Estado de Cambios en el Capital de Trabajo (Fondo de Maniobra).

Así por ejemplo, Anthony y Reece ${ }^{1}$ expresan que el Estado de Flujo de Caja explica el cambio en la cuenta Caja/Bancos y el Estado de Flujo de Fondos explica los cambios que se producen en el Capital de Trabajo. Block y Hirt ${ }^{2}$ anotan que el Estado de Origen y Aplicación de Recursos mide los cambios en las partidas del Balance General a través de un periodo de tiempo, en tanto que un Estado de Origen y Aplicación de Recursos Modificado que incorpora conciliaciones en las partidas depreciación y utilidades acumuladas, corresponde a un enfoque de capital de trabajo. Ross y otros ${ }^{3}$ resaltan que el capital de trabajo neto es el efectivo más los demás elementos del capital de trabajo, añadiendo que el Estado de Origen y Aplicación de Fondos muestra todas las transacciones que afectan la disposición de efectivo de la empresa.

En realidad, dependiendo del objetivo del análisis, su construcción permitirá conocer los recursos económicos con que cuenta la empre- sa, o establecer los cambios en el Fondo de Maniobra, o bien explicar el cambio observado en el efectivo, poniendo énfasis en las interrelaciones existentes en tres áreas de decisiones financieras: inversión, financiamiento y distribución de utilidades.

En la gestión financiera como parte integral de los costos históricos, los Estados de Movimiento de Fondos permiten describir lo ocurrido con los fondos aportados por las operaciones comerciales del negocio vía utilidades, los fondos aportados por los accionistas, por la obtención de los préstamos, por la emisión de bonos o por la venta de activos fijos y financieros.

Este instrumento proporciona la información para responder a ciertas interrogantes: ¿cómo se ha logrado un aumento o disminución del Fondo de Maniobra?, ¿se ha impactado el nivel de Fondo de Maniobra por retención de utilidades, por aporte de accionistas, por aumento o disminución de préstamos, por adquisición o venta de activos fijos e inventarios, por distribución de utilidades vía dividendos? La información que proporciona permite apreciar la tendencia en los cambios del capital de trabajo, la necesidad de financiar aumentos permanentes de dicho capital y el mantenimiento de la capacidad productiva.

En resumen, proporciona la clave de la forma en que la empresa está manejando y financiando su inversión en ella misma. Por todo lo anotado este reporte es dinámico y útil en la gestión de la gerencia.

En este contexto, el presente trabajo tiene como propósito integrar los diferentes conceptos y resaltar las bondades de este instrumento, para su apropiada comprensión e interpretación que permitirá conocer lo sucedido en el manejo de los fondos en el pasado y los intentos para formular el pronóstico financiero, a efectos de conocer la futura jerarquía del efectivo como variable determinante en el establecimiento del valor de la empresa desde el punto de vista de las finanzas.

El trabajo se estructura en tres partes. En esta primera parte se procederá a presentar la conceptualización vinculada a las diversas modalidades de presentación de dicho estado financiero en función de la definición de los fondos, así como los procedimientos más usados para prepararlo. Finalmente se procederá a su confección tal como lo exige la Comisión Nacio- 
nal Supervisora de Empresas y Valores (CONASEV) en su Resolución No 182 , señalando que las empresas deberán incluir el Estado de Flujos de Efectivo en sustitución del Estado de Cambios en la Situación Financiera.

En la segunda parte se presentarán las interrelaciones existentes entre las diversas partidas del Balance y del Estado de Resultados (EPG) con el Estado de Movimiento de Fondos, para un preciso entendimiento de sus cambios e interpretación adecuada; de modo tal que se aprecie la bondad del conjunto como herramienta de análisis y toma de decisiones gerenciales. Para una explicación numérica, en ambas partes se hace uso de información que corresponde a una empresa manufacturera.

En la tercera parte se interrelacionan los conceptos analizados en las dos primeras partes, pero aplicados con información numérica para el caso de una empresa comercial.

\section{¿CUÁL ES EL CONCEPTO DE FONDOS?}

En la literatura de la Contabilidad y Finanzas, dependiendo de como se presentan los cambios específicos en el Balance, en el proceso de establecer el Estado de Movimiento de Fondos, tradicionalmente se adoptan tres enfoques de definición de fondos ${ }^{4,5}$, tal como sigue:

a) Valores económicos o recursos totales. Enfoque que señala que los recursos provienen de los accionistas y acreedores, y aplicados en el lado de los activos, se traduce en la composición de la inversión de los recursos. Financieramente hablando los fondos son los recursos con que cuenta la empresa para destinarlos a las necesidades propias de sostenimiento y crecimiento del negocio.

b) El Fondo de Maniobra. Conocido tradicionalmente como Capital de Trabajo ha sido usado ampliamente pero en un sentido contable, como la diferencia entre el activo corriente menos el pasivo corriente. Se apreciará posteriormente que representa un concepto financiero más profundo y útil para decisiones de gerencia.

c) Caja o Efectivo. Enfoque en el cual el movimiento de fondos es equivalente a la corriente de ingresos y egresos (desembolsos) a partir del movimiento de las diferentes cuentas del Balance, con impacto directo en la cuen- ta Caja/Bancos. Frecuentemente se define estos fondos como liquidez.

De manera general la conceptualización financiera de fondos implica el concepto de dinero, como también el equivalente de dinero es caja o efectivo.

Entonces es fundamental que para la formulación y comprensión del Estado de Movimiento de Fondos debe conocerse la definición e implicancia de cada una de las alternativas anotadas, y su utilización estará en concordancia con el objetivo del análisis.

Su adecuada y rigurosa comprensión conducirá a una correcta interpretación de la gestión, cuando se hace uso de los estados financieros como herramientas de la administración gerencial.

Los Estados de Movimiento de Fondos se consideran estados dinámicos dado que describen cambios durante un periodo de tiempo. Los cambios no son un stock; representa un flujo y por tanto se consignan en el cuadro de flujo de fondos que revela las decisiones financieras con implicancias a largo plazo.

\section{FONDOS COMO RECURSOS TOTALES (FLUJO DE FONDOS)}

Para explicar esta posición, la base es la ecuación contable (Cuadro 1):

Cuadro 1. Activos $=$ Pasivo + Patrimonio

\begin{tabular}{|cc|}
\hline ACTIVOS & PASIVO + PATRIM. \\
& \\
AC & PC \\
AF & PLP \\
AI & PAT \\
Total Activos & Total Pasivos + Pat. \\
USO DE FONDOS & FUENTE DE FONDOS \\
(APLICACIONES) & (ORIGEN) \\
& (Participaciones) \\
\hline
\end{tabular}


El análisis del movimiento de fondos tradicionalmente ha sido conceptuado como los cambios en el balance de una empresa durante un periodo de tiempo, basándose en una definición de fuentes (activos que disminuyen y pasivos que aumentan) y de usos (activos que aumentan y pasivos que disminuyen).

Los activos totales se conceptúan como uso de fondos y las participaciones totales como fuente de estos fondos. Los aumentos en los activos totales se explican por los aumentos en las participaciones, que pueden deberse a aumentos de los fondos por las operaciones propias del negocio. Un aumento en cualquier activo puede deberse también a una disminución en otro activo; similarmente un aumento en una cuenta de participaciones puede significar una reducción de una partida de participaciones.

En la formulación de este esquema, la manera práctica tradicional de obtenerlo es teniendo en cuenta la diferencia de las cuentas del Balance Inicial y Final para un periodo determinado, resultando lo siguiente (Cuadro 2):

Cuadro 2. Esquema de fondos como recursos totales.

Entonces, en la definición de fondos en

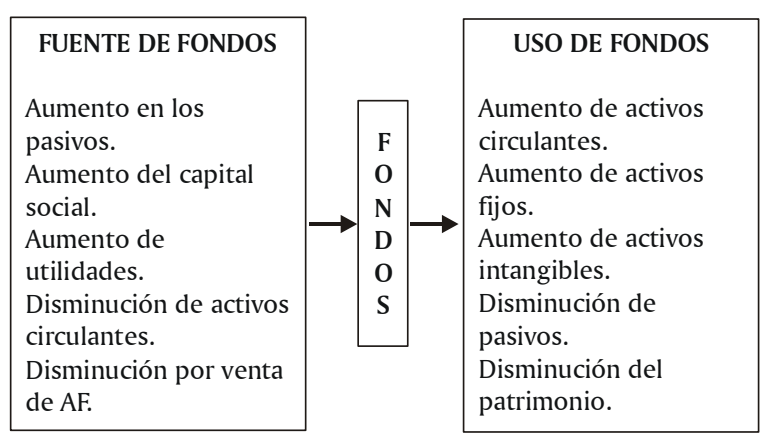

términos de activos totales y participaciones totales, el estado de movimiento de fondos se centra en los cambios de todas las cuentas del Balance entre dos fechas diferentes en el tiempo.

Para una mejor apreciación de dicho esquema tradicional, el Estado de Movimiento de Fondos se ha determinado sobre la base de la información numérica de la empresa Industrial Textil S.A., que se señala en el Anexo N. ${ }^{\circ} 1$.

Veamos el siguiente cuadro (Cuadro N. ${ }^{\circ} 3$ ):
Cuadro 3. Fuentes y usos de fondos en dólares.

\begin{tabular}{|lr|}
\hline \multicolumn{2}{|c|}{ Fuente de Fondos } \\
Utilidades del ejercicio & 50000 \\
Depreciación & 50000 \\
Sobregiros & 10000 \\
Documentos por pagar & 30000 \\
Cuentas por pagar (proveedores) & 30000 \\
Gastos pagados por adelantado & 10000 \\
Pasivos a largo plazo & 20000 \\
Total Fuentes & 200000 \\
\hline & \\
Caja y Bancos Uso de Fondos & 10000 \\
Cuenta por cobrar (clientes) & 30000 \\
Existencias & 50000 \\
Gastos devengados por pagar & 10000 \\
Edificaciones y Planta & 100000 \\
Total Usos & 200000 \\
\hline
\end{tabular}

Este cuadro de flujo de fondos elaborado como resultado de la diferencia de todas las cuentas del Balance, es cuestionado por los analistas financieros en la medida que privilegia las anotaciones contables, registrando en forma simplista la utilidad retenida y la depreciación como fuente de fondos; omitiendo u ocultando ciertas decisiones financieras como la distribución de dividendos en el caso del ejemplo.

Actualmente se realizan ajustes para presentar un cuadro de flujo de fondos que transmite información más relevante como producto de las decisiones de gestión, por cuanto incorpora los conceptos de Fondos Generados por Operaciones (FGO), variación del Fondo de Maniobra, los cambios de los recursos permanentes e inmovilizados; recomendándose además incorporar cuentas derivadas de decisiones que no forman parte de las operaciones corrientes normales, como por ejemplo la venta de activos fijos que se debe mostrar como una fuente de fondos. El cuadro modificado (Cuadro 4) es el que sigue:

Cuadro 4. Fuentes y usos de fondos en dólares (al 31.12.03).

\begin{tabular}{|c|c|}
\hline Fuente de Fondos & \\
\hline Fondos Generados por Operaciones (FGO) & 160500 \\
\hline Pasivos a largo plazo & 20000 \\
\hline Total Fuentes & 180500 \\
\hline \multicolumn{2}{|l|}{ Uso de Fondos } \\
\hline Aumento Fondo de Maniobra & 10000 \\
\hline Edificaciones y Planta & 100000 \\
\hline Dividendos & 60500 \\
\hline Total Usos & 180500 \\
\hline
\end{tabular}


La información que proporciona el último cuadro es más explicativa: los fondos que se derivan de las operaciones comerciales contribuyen decididamente con el $89 \%$ en el sostenimiento de la ampliación en planta que consume el $56 \%$ de los recursos, la distribución de dividendos el 33\% y el aumento del Fondo de Maniobra el $11 \%$.

\section{FONDOS COMO FONDO DE MANIOBRA}

En la contabilidad tradicional se ha definido los fondos como Capital de Trabajo, denominado modernamente Fondo de Maniobra (FM). Para explicar este concepto, es necesario representar el Balance como se ilustra en el Cuadro 5:

Cuadro 5. Esquema Conceptual del FM.

\begin{tabular}{|c|c|c|}
\hline \multirow{2}{*}{$\begin{array}{l}\qquad \text { ACTIVO } \\
\text { Activo Circulante (AC) } \\
\text { (Activo Corriente) }\end{array}$} & & \multirow{5}{*}{$\begin{array}{l}\text { Recursos Permanentes (RP) } \\
\text { 1) Pasivo Largo Plazo } \\
\text { 2) Patrimonio (capital } \\
\text { social más utilidades } \\
\text { retenidas) }\end{array}$} \\
\hline & & \\
\hline \multirow{3}{*}{$\begin{array}{l}\text { Inmovilizaciones (AI) } \\
\text { 1) Activo Fijo } \\
\text { 2) Activo Intangible }\end{array}$} & FM & \\
\hline & & \\
\hline & & \\
\hline
\end{tabular}

Entonces la conceptualización del Fondo de Maniobra presenta dos vertientes:

$\mathrm{FM}=\mathrm{AC}-\mathrm{PC}$, concepto tradicional.

$\mathrm{FM}=\mathrm{RP}-\mathrm{AI}$, concepto moderno útil para la gerencia financiera.

Donde: $\mathrm{FM}=$ Fondo de Maniobra.

Por eso es necesario precisar la definición de Fondo de Maniobra (FM) como la diferencia aritmética del AC menos PC, que se relaciona con un concepto de inversión, vale decir como uso del capital permanente; en tanto que en la definición alternativa de RP-AI, el FM se entiende como una fuente financiera estructural, resultado de la diferencia de los recursos permanentes del Balance, es decir, las fuentes de capital permanentes menos los activos inmovilizados, mostrando qué proporción se usa de dicho capital permanente, o bien qué proporción del activo está inmovilizado en diversas partidas.

Al desarrollar parte del análisis financiero de una empresa se espera determinar su capacidad de pago actual frente a sus acreedores, si- tuación que esta íntimamente vinculada a la magnitud del FM que se exhibe en el Balance como un stock, pero la variación o cambio del FM representa un flujo y se exhibe como flujo de fondos. Ambos conceptos son sumamente importantes para una adecuada comprensión de este tema.

Complementando el análisis en la definición de fondos como FM, todo el Balance se divide en dos partes que son equivalentes en la cuantía de los cambios totales, como se presenta y explica a continuación en el Cuadro 6:

Cuadro 6. Esquema del FM: Visión en el Corto y Largo Plazo.

\begin{tabular}{|c|c|c|}
\hline ACTIVO & PASIVO + PAT & CONCEPTUALIZACIÓN \\
\hline $\begin{array}{l}\text { Activo } \\
\text { Circulante }\end{array}$ & $\begin{array}{l}\text { Pasivo } \\
\text { Circulante }\end{array}$ & $\begin{array}{l}\text { Resumen de cambios en } \\
\text { el FM (movimiento de } \\
\text { fondos a corto plazo). }\end{array}$ \\
\hline $\begin{array}{l}\text { Inmovilizados: } \\
\text { A. Fijo } \\
\text { A. Intangible }\end{array}$ & $\begin{array}{l}\text { Recursos Perm. } \\
\text { Pasivo LP } \\
\text { Patrimonio }\end{array}$ & $\begin{array}{l}\text { Estado de Movimiento de } \\
\text { Fondos explicando los } \\
\text { cambios en el FM } \\
\text { (movimiento de fondos } \\
\text { a largo plazo). }\end{array}$ \\
\hline
\end{tabular}

a) La parte superior del cuadro denominado también estructura patrimonial circulante muestra el resumen de cambios del Fondo de Maniobra, como movimiento de fondos de las cuentas del circulante para obtener el incremento o reducción en el Capital de Trabajo, que representa una repercusión en el corto plazo. Explica el contenido interno del cambio en el Fondo de Maniobra.

Las transacciones que se realizan entre partidas del mismo circulante no tiene efecto alguno sobre los fondos proporcionados o usados, por lo que no afectan la cantidad de fondos del Fondo de Maniobra, solo cambian su composición. Estas transacciones no se registran como fuente ni como uso de fondos en la medida que configuran un reordenamiento de las cuentas que en conjunto forman el Fondo de Maniobra.

b) La parte inferior denominada también estructura patrimonial permanente, presenta el Estado de Movimiento de Fondos de las cuentas del inmovilizado y de recursos permanentes, razón que permite apreciar que sus repercusiones son más a largo plazo. Los cambios en la parte inferior del Balance se analizan para explicar los cambios en la parte su- 
perior, dado que exhibe las causas de los aumentos y reducciones en el Fondo de Maniobra. Bajo este concepto, el Estado de Movimiento de Fondos grafica un esquema del financiamiento a largo plazo de la empresa, en la medida que se han eliminado los movimientos financieros a corto plazo.

Este esquema permite responder determinadas interrogantes como ¿cuál es la proporción del financiamiento a largo plazo por nuevas deudas o aporte dinerario de accionistas o por retención de utilidades o por reducción del Fondo de Maniobra?, ¿qué proporción del total de fondos disponibles se usó para adquisición de activos fijos y mejoras en planta, pago de deudas a largo plazo o aumento del Fondo de Maniobra?

c) Las transacciones contables relevantes que modificará el Fondo de Maniobra son aquellas que una mitad del asiento afecta la parte superior del Balance y la otra mitad la parte inferior. Ejemplo: equipos vs. efectivo; caja vs. capital social, etc. La transacción que se mantiene solo en uno de los bloques del Balance no tendrá efecto alguno sobre los fondos proporcionados o aplicados, por lo que no deben aparecer en un Estado de Movimiento de Fondos. Ejemplo: cuentas por cobrar vs. caja; sobregiros vs. proveedores. d) Se generan fondos, lo que implica un incremento del Fondo de Maniobra cuando las operaciones realizadas con partidas de la estructura patrimonial permanente, al afectar también partidas de la estructura patrimonial circulante, implican un incremento del valor neto de dicha estructura circulante como Fondo de Maniobra.

Se produce una aplicación de fondos, lo que implica una reducción del Fondo de Maniobra, cuando partidas de la estructura patrimonial circulante, implican una reducción del valor neto de dicha estructura circulante como Fondo de Maniobra.

Entonces, en la definición de fondos en términos de Fondo de Maniobra, el estado de movimiento de fondos explica los cambios que se producen en el Fondo de Maniobra y en las cuentas de recursos permanente y activos inmovilizados del Balance entre dos fechas diferentes en el tiempo.

Para aplicar el enfoque citado y asegurar su entendimiento se procesa la información del Anexo $\mathrm{N}^{\circ} 1$, resaltando las dos partes del Balance que se complementan mutuamente, mostrando los resultados en el Cuadro 7:

Cuadro 7. Hoja de Trabajo del Flujo de Fondos en dólares

\begin{tabular}{|c|c|c|c|c|}
\hline CONCEPTO & 2002 & 2003 & \multicolumn{2}{|c|}{ CAMBIOS FONDO DE MANIOBRA } \\
\hline ACTIVO CIRCULANTE & & & Aumentos & Disminuciones \\
\hline Caja y bancos & 30000 & 40000 & 10000 & 0000 \\
\hline Clientes & 170000 & 200000 & 30000 & 0000 \\
\hline Existencias & 130000 & 180000 & 50000 & 0000 \\
\hline Otros activos & 40000 & 30000 & 0000 & 10000 \\
\hline TOTAL AC & 370000 & 450000 & 90000 & 10000 \\
\hline \multicolumn{5}{|l|}{ PASIVO CIRCULANTE } \\
\hline Sobregiros & 20000 & 30000 & 0000 & 10000 \\
\hline Proveedores & 50000 & 80000 & 0000 & 30000 \\
\hline Otros pasivos & 80000 & 100000 & 0000 & 20000 \\
\hline TOTAL PC & 150000 & 210000 & 0000 & 60000 \\
\hline TOTAL aumento (Reducción) & & & 90000 & 70000 \\
\hline CAMBIO NETO EN EL FM & & & & 20000 \\
\hline Concepto & & & Fuentes & Usos \\
\hline \multicolumn{5}{|l|}{ INMOVILIZADOS } \\
\hline Activo fijo bruto & 1000000 & 1100000 & & 100000 \\
\hline (-) Depreciación acumulada & 550000 & 600000 & 50000 & \\
\hline TOTAL inmovilizados & 450000 & 400000 & & 50000 \\
\hline \multicolumn{5}{|l|}{ RECURSOS PERMANENTES } \\
\hline Pasivo LP & 70000 & 90000 & 20000 & \\
\hline Capital social & 400000 & 400000 & & \\
\hline Utilidades retenidas & 250000 & 300000 & 50000 & \\
\hline TOTAL recursos permanentes & 720000 & 790000 & 70000 & \\
\hline Total fuentes y usos & & & 70000 & 50000 \\
\hline \multicolumn{4}{|c|}{$\begin{array}{l}\text { FONDOS APLICADOS PARA INCREMENTAR EL FM } \\
\text { (exceso de fuentes sobre usos) }\end{array}$} & 20000 \\
\hline
\end{tabular}


Luego, el reporte formal de movimiento de fondos en este enfoque, aplicando los conceptos enunciados, se visualiza en el Cuadro 8:

\begin{tabular}{|l|l|l|}
\hline \multicolumn{3}{|c|}{$\begin{array}{c}\text { Cuadro 8. Estado de movimiento de fondos } \\
\text { (Fondos como Fondo de Maniobra) }\end{array}$} \\
\hline $\begin{array}{l}\text { Fondos Proporcionados: } \\
\text { Por aumento de PLP } \\
\text { Por aumento de utilidades retenidas }\end{array}$ & 20000 & 70000 \\
Fondos Usados: & 50000 & 50000 \\
Al aumento de activos fijos (Planta) & 50000 & 20000 \\
$\begin{array}{l}\text { Exceso de fondos proporcionados sobre } \\
\text { fondos usados. (Fondos Aplicados para } \\
\text { aumentar el Fondo de Maniobra) }\end{array}$ & \\
\hline
\end{tabular}

Vale recalcar que el cuadro informa de las necesidades de recursos a largo plazo en el período y la forma cómo se financió (deuda a largo plazo, emisión de acciones y/o bonos, utilidades retenidas) o también los recursos que pueden provenir de venta de activos fijos o por una disminución del capital circulante. En el ejemplo, muy por el contrario, el cambio ha producido un aumento del Fondo de Maniobra.

Sin embargo, para propósitos de análisis financiero el cuadro de flujo de fondos debe mostrar en forma explícita los resultados de las decisiones financieras, como es el caso del reparto de dividendos que no se muestra en el cuadro anterior. Por esta razón, manteniendo invariables los conceptos centrales de la explicación en la formación del cambio del Fondo de Maniobra, se presenta el cuadro modificado (Cuadro 9) que señala:

\begin{tabular}{|c|c|c|}
\hline \multicolumn{3}{|c|}{ Cuadro 9. Flujo de Fondos Modificado } \\
\hline Utilidad neta & 110500 & \\
\hline $\begin{array}{c}\text { Mas depreciación y } \\
\text { amortizaciones }\end{array}$ & 50000 & \\
\hline $\begin{array}{l}\text { Fondos Generados por } \\
\text { Operaciones (FGO) (1) }\end{array}$ & & 160500 \\
\hline $\begin{array}{l}\text { Otras fuente de fondos } \\
\text { No Circulantes: (2) }\end{array}$ & & 20000 \\
\hline Aumento Pasivo a largo plazo & 20000 & \\
\hline $\begin{array}{c}\text { Aumento Capital social } \\
\text { TOTAL FUENTE DE FONDOS } \\
(3=1+2)\end{array}$ & 00000 & 180500 \\
\hline $\begin{array}{l}\text { APLICACIÓN DE FONDOS } \\
\text { No Circulantes : }\end{array}$ & & 160500 \\
\hline Aumento Activos fijos & 100000 & \\
\hline Dividendos repartidos & 60500 & \\
\hline $\begin{array}{c}\text { Aumento (Reducción) FONDO DE } \\
\text { MANIOBRA }\end{array}$ & & 20000 \\
\hline
\end{tabular}

\section{FONDOS EXPRESADOS COMO LIQUIDEZ} (ESTADO DE FLUJOS DE EFECTIVO)

Con este enfoque se trata de apreciar los fondos en términos de movimiento de caja, descartando las cuentas que no involucren ingresos y egresos de dinero, registrándose tres partes en este esquema. En efecto, el documento Estado Flujos de Efectivo según señala la CONASEV $^{6}$, muestra el efecto de los cambios de efectivo y equivalentes de efectivo en un periodo determinado, debiendo mostrar separadamente lo siguiente:

a) Actividades de Operaciones. Corresponde a las actividades derivadas de las operaciones propias del negocio: comprar, producir y vender.

En esta parte se reflejan los flujos de efectivo usados u obtenidos de actividades de operación, que corresponden a las transacciones en efectivo y otras partidas que intervienen en la formación de la utilidad neta; vale decir, las partidas que forman parte del Estado de Resultados (EPG).

Las cuentas de ingresos y egresos son cobranza por venta, pago por materias primas, suministros y remuneraciones, pago por diversos servicios de terceros, pago y cobranza por intereses, pago por impuestos y contribuciones sociales, entre otros.

b) Actividades de Inversión. Son los fondos obtenidos o aplicados por decisiones de inversión, tales como compra o venta de activos fijos, compra o venta de instrumentos financieros, compra o venta de patentes o franquicias, entre otros.

c) Actividades de Financiamiento. Son los fondos obtenidos o aplicados por gestiones financieras o decisiones de financiamiento, tales como gestionar o recibir préstamos o cancelarlos, recibir aportes de accionistas, emisión de bonos, distribuir utilidades vía dividendos.

Dicho estado financiero debe informar sobre el efectivo neto obtenido y usado por las actividades de operación, inversión y financia-miento y el efecto neto de esos flujos. El efectivo al inicio y final del periodo debe considerar el aumento o disminución de efectivo del periodo, que debe ser equivalente a dicho efecto neto de los flujos. 
Una forma alternativa de presentar los cambios en un ejercicio en el Balance General y el EPG en las referidas tres actividades, excluyendo las partidas contables no desembolsables, se muestra en el Cuadro 10:

\begin{tabular}{|c|c|c|c|}
\hline \multicolumn{4}{|c|}{$\begin{array}{l}\text { Cuadro 10. Movimiento de fondos en términos de liquidez en } \\
\text { dólares. }\end{array}$} \\
\hline $\begin{array}{l}\text { Concepto / Actividad } \\
\text { Ingresos de Caja } \\
\text { (FUENTES) } \\
\text { Inversiones en valores }\end{array}$ & $\begin{array}{l}\text { Operación } \\
2080000\end{array}$ & Inversión & $\begin{array}{l}\text { Financiamiento } \\
20000\end{array}$ \\
\hline Documentos por pagar & 30000 & & \\
\hline $\begin{array}{l}\text { Gastos pagados por } \\
\text { adelantado }\end{array}$ & 10000 & & \\
\hline Sobregiros & 10000 & & \\
\hline $\begin{array}{l}\text { Cuentas por pagar } \\
\text { (proveedores) }\end{array}$ & 30000 & & \\
\hline Pasivo a largo plazo & & & 20000 \\
\hline Ventas & 2000000 & & \\
\hline Egresos de Caja (USOS) & 1929500 & 100000 & 60500 \\
\hline $\begin{array}{c}\text { Gastos devengados por } \\
\text { pagar }\end{array}$ & 10000 & & \\
\hline Existencias & 50000 & & \\
\hline $\begin{array}{l}\text { Cuentas Por cobrar } \\
\text { (clientes) }\end{array}$ & 30000 & & \\
\hline Edif., y Planta (Bruto) & & 100000 & \\
\hline Costo de venta & 1500000 & & \\
\hline $\begin{array}{l}\text { Gastos de venta y } \\
\text { administrativos }\end{array}$ & 230000 & & \\
\hline Gastos por intereses & 20000 & & \\
\hline Impuesto a la renta & 89500 & & \\
\hline Dividendos & & & 60500 \\
\hline Saldo de Caja Neto & 150500 & $(100000)$ & $(40500)$ \\
\hline
\end{tabular}

Luego, como resultado del movimiento de fondos expresado como movimientos de caja, se tiene el Cuadro 11:

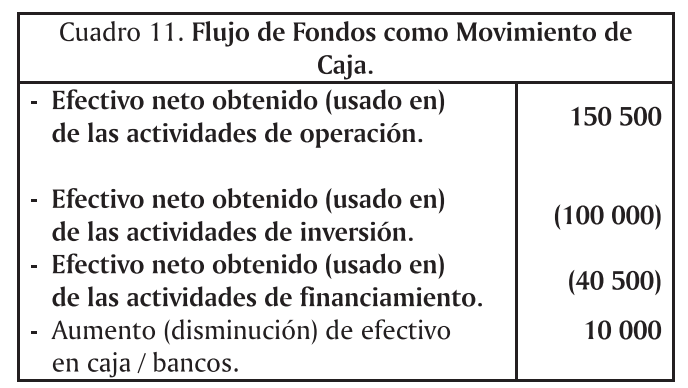

Visto desde el concepto de ingresos y egresos en el ejemplo para el periodo 2003, se tiene el esquema señalado en el Cuadro 12:

\begin{tabular}{|c|c|c|c|c|c|}
\hline \multicolumn{6}{|c|}{ Cuadro 12. Flujo de Ingresos y Egresos (en dólares). } \\
\hline \multicolumn{3}{|c|}{ Ingresos (Fuentes) } & \multicolumn{3}{|c|}{ Egresos (Aplicaciones) } \\
\hline Operación & 150500 & $100,00 \%$ & Inversión & 100000 & 66,50 \\
\hline & & & Financiamient & 40500 & 27,00 \\
\hline & & & $\begin{array}{l}\text { Aumento de } \\
\text { Caja Bancos }\end{array}$ & 10000 & $06,50 \%$ \\
\hline Total & 150500 & $100,00 \%$ & Total & 150500 & $100,00 \%$ \\
\hline
\end{tabular}

Del cuadro anterior se observa que las actividades de inversión y financiamiento, así como el incremento de liquidez que se refleja en la cuenta Caja /Bancos, se ha debido solo a la contribución de los ingresos netos generados por la actividad de operación del negocio.
En el Cuadro 13 se presenta el movimiento de fondos en términos de liquidez (efectivo), expresados bajo la forma de Fondos Generados por Operaciones (FGO), Caja Generada por Operaciones (CGO) y las fuentes y usos de caja por inversiones y financiamiento permanente.

\begin{tabular}{|c|c|c|}
\hline \multicolumn{3}{|c|}{ Cuadro 13. Flujo de Fondos en Términos de Liquidez. } \\
\hline CONCEPTO & & Totales \\
\hline Utilidad Neta (UN) & 110500 & \\
\hline (más) Depreciación y amortización & 50000 & \\
\hline $\begin{array}{l}\text { Fondos Generados por Operaciones } \\
\text { (FGO) (1) }\end{array}$ & & 160500 \\
\hline $\begin{array}{l}\text { Variación en la Necesidad (2) } \\
\text { Operativa de Fondos (NOF) }\end{array}$ & & $(10000)$ \\
\hline (menos) Aumento de clientes & $(30000)$ & \\
\hline (menos) Aumento de existencias & $(50000)$ & \\
\hline (más) Aumento de proveedores & 30000 & \\
\hline (más) Aumento otros APC & 40000 & \\
\hline $\begin{array}{l}\text { Caja Generada por Operaciones (CGO) } \\
(3=1+2)\end{array}$ & & 150500 \\
\hline FUENTES DE CAJA NO Circulantes (4) & & 20000 \\
\hline Aumento de préstamo de largo plazo & 20000 & \\
\hline Aumento del capital social & 00000 & \\
\hline TOTAL FUENTES DE CAJA $(5=3+4)$ & & 170500 \\
\hline $\begin{array}{c}\text { APLICACIONES DE CAJA NO } \\
\text { Circulantes (6) }\end{array}$ & & 160500 \\
\hline Aumento de Edificaciones y Planta & $(100000)$ & \\
\hline Dividendos entregados & $(60500)$ & \\
\hline $\begin{array}{l}\text { Aumento (Reducción) }(7=5-6) \\
\text { en CAJA / BANCOS }\end{array}$ & & 10000 \\
\hline Caja inicial (principios de 2003) & & 30000 \\
\hline CAJA FINAL (fines de 2003) & & 40000 \\
\hline
\end{tabular}

Es importante resaltar que el cargo por depreciación tan solo constituye un asiento contable y no implica directamente un movimiento de fondos. Para transformar un flujo contable a un flujo de fondos se añade la deducción no desembolsable por US\$50 000 correspondiente en este caso a la depreciación la cual aparecía restando en el Estado de Resultados (EPG). En consecuencia, sumar la depreciación a la utilidad sólo representa una operación aritmética. Enfatizando: la depreciación y amortización de intangibles no constituyen una fuente de fondos, pero sí proporciona beneficios de ahorro de impuestos ${ }^{7}$.

Se recomienda observar detenidamente y tomar el cuadro anterior como una guía práctica para la presentación del flujo de fondos en términos de liquidez. El rubro denominado aquí como Necesidad Operativa de Fondos (NOF) solo debe incluir de manera explícita (por ser muy relevante para el análisis), las cuentas del circu- 
lante que forma la triada clientes/existencia/proveedores, añadiendo una cuenta denominada Otros APC, que comprende las restantes cuentas del activo y pasivo corriente, tal como sigue para el ejemplo anotado:

\begin{tabular}{|c|r|}
\hline \multicolumn{2}{|c|}{ Cuadro 14. Cuenta Otros APC. } \\
\hline Concepto & Monto en dólares \\
\hline $\begin{array}{c}\text { Más disminución gastos } \\
\text { pagados por adelantado }\end{array}$ & $\mathbf{1 0 0 0 0}$ \\
Más aumento sobregiros \\
Más aumento documentos \\
$\begin{array}{c}\text { por pagar } \\
\text { Menos disminución gastos } \\
\text { devengados por pagar }\end{array}$ & $\mathbf{1 0 0 0 0}$ \\
Otros APC & $\mathbf{3 0 0 0 0}$ \\
\hline
\end{tabular}

Con este enfoque, en la definición de fondos en términos de liquidez, el movimiento de fondos se convierte en movimiento de liquidez; las entradas de caja son las FUENTES y las salidas de caja son los USOS entre un período determinado, dando como resultado el cambio en la cuenta Caja/Bancos del Balance.

\section{CONCLUSIÓN}

En la medida que se ponga a disposición de profesionales y empresarios la utilidad práctica de las herramientas metodológicas, se estará facilitando su uso y mejor manejo en la solución de problemas; en este caso particular en el diseño y solución de problemas vinculados al manejo del movimiento de fondos.

\section{NOTAS}

1 Anthony, R. y Reece, J. Principios Contables. 3ra Edición Argentina, El Ateneo Editorial, 1982

2 Block, S. y Hirt, G. Fundamentos de Administración Financiera. Sexta reimpresión. México, Editorial Continental, 1994.

3 Ross, S., Westerfield, R. y otros. Finanzas Corporativas. Quinta Edición, México, Mc Graw Hill, 2000.

4 Pronosticación Financiera Básica (Adaptado del Harvard Business School, 1954). ESAN, CO. 245.

5 Los Movimientos de Fondos por Josué Cárdenas. ESAN, Doc. F 3191.

6 Frank, H. El Flujo de Efectivo. Revista Proyección de AGESAN Sección Profesional), Setiembre 1997.

7 Block, S. y Hirt, G. Fundamentos de Administración Financiera. Op. cit., pp. 61.

\begin{tabular}{|c|c|c|c|}
\hline Concepto & $\mathrm{Al} 31.12 .02$ & $\mathrm{Al} 31.12 .03$ & Variación 03-02 \\
\hline \multirow{2}{*}{\multicolumn{4}{|c|}{$\begin{array}{l}\text { Activos } \\
\text { Activos circulantes }\end{array}$}} \\
\hline & & & \\
\hline $\begin{array}{l}\text { Caja y bancos } \\
\text {. }\end{array}$ & $\begin{array}{l}30000 \\
10000\end{array}$ & $\begin{array}{l}40,000 \\
10,000\end{array}$ & 10,000 \\
\hline $\begin{array}{l}\text { Cuentas por cobrar } \\
\text { (clientes) }\end{array}$ & 170000 & 200,000 & 30,000 \\
\hline Existencias & 130000 & 180,000 & 50,000 \\
\hline Gastos pagados por & 30000 & 20,000 & $(10,000)$ \\
\hline Total AC & 370000 & 450,000 & \\
\hline Activos fijos & & & \\
\hline Edificaciones y planta & 1000000 & 1100000 & 100000 \\
\hline $\begin{array}{l}\text { - (Depreciación } \\
\text { acumulada) }\end{array}$ & 550000 & 600000 & 50000 \\
\hline $\begin{array}{c}\text { Edificaciones y planta } \\
\text { (Neto) }\end{array}$ & 450000 & 500000 & \\
\hline Total activos fijos & 450000 & 500000 & \\
\hline Inversiones permanentes & 50000 & 50000 & \\
\hline Total activos & 870000 & 1000000 & \\
\hline \multirow{2}{*}{\multicolumn{4}{|c|}{$\begin{array}{l}\text { Pasivo y patrimonio } \\
\text { Pasivo circulante }\end{array}$}} \\
\hline & & & \\
\hline Sobregiros & 20000 & 30000 & 10000 \\
\hline $\begin{array}{l}\text { Cuentas por pagar } \\
\text { (proveedores) }\end{array}$ & 50000 & 80000 & 30000 \\
\hline Documentos por pagar & 40000 & 70000 & 30000 \\
\hline $\begin{array}{l}\text { Gastos devengados por } \\
\text { pagar }\end{array}$ & 4000 & 30000 & $(10000)$ \\
\hline Total PC & 150000 & 210000 & \\
\hline Pasivo a largo plazo (PLP) & 70000 & 90000 & 20000 \\
\hline Total pasivo & 220000 & 300000 & \\
\hline Patrimonio & & & \\
\hline Capital social & 400000 & 400000 & \\
\hline Utilidades retenidas & 250000 & 300000 & 50000 \\
\hline Total Patrimonio & 650000 & 700000 & \\
\hline Total pasivo y & 870000 & 1000000 & \\
\hline
\end{tabular}

\section{Estado de resultados 2003 en dólares}

\begin{tabular}{|l|r|}
\hline Ventas & 2000000 \\
Costo de ventas & 1550000 \\
Utilidad bruta & 450000 \\
Gastos de venta y administración & 230000 \\
Utilidad de operación & 220000 \\
Gastos financieros & 20000 \\
Utilidad antes de impuestos (UAI) & 200000 \\
Impuesto a la renta & 89500 \\
Utilidad después de impuestos (U. Neta) & 110500 \\
Dividendos & 60500 \\
Utilidades retenidas & 50000 \\
& \\
Anotaciones: & \\
Depreciación 2003 (incluido en el costo de venta) & 50000 \\
Gastos de Explotación (desembolsables) & \\
Gastos de venta y administración & 230000 \\
Gastos financi eros & 20000 \\
Impuesto a la renta & 89500 \\
Total gastos de explotación (desembolsables) & 339500 \\
\hline
\end{tabular}

ANEXO 1. Industrial Textil S.A. 
